\title{
MANUAL DE INTERVENCIÓN EN DILEMAS IMPLICATIVOS
}

\author{
Joana Senra \\ Universidade do Minho \\ Guillem Feixas \\ Universitat de Barcelona \\ Eugénia Fernandes \\ Universidade do Minho
}

\begin{abstract}
The notion that human beings face internal conflicts is very old in the field of psychotherapy. Also, it is common the idea that symptoms could be derived from those conflicts. However, attempts for developing ways of appraising those conflicts so that they can be measured and tested empirically are almost inexistent. Precisely, the MultiCentre Dilemma Project is aimed at investigating the role of those conflicts, termed implicative dilemmas or dilemmatic constructs, in health using the Repertory Grid Technique as a method to identify them. So far, a higher presence of those conflicts has been found in a variety of clinical problems (depression, social phobia, somatic problems, etc.) in comparison to non-clinical samples. Therefore, it seems convenient to develop a form of intervention aimed to dealing and resolving these conflicts.

In this paper a therapy manual focused on implicative dilemmas resolution is presented. It consists of a structured intervention for 15 sessions, designed mainly for research and training in psychotherapy, and based on Personal Construct Psychotherapy.

Key words: Personal constructs; dilemmas; pychotherapy; Repertory Grid
\end{abstract}

\section{INTRODUCCIÓN}

En este artículo se presenta un manual de intervención enfocado a la resolución de dilemas implicativos, tal como son identificados a través de la Técnica de Rejilla (TR). Se trata de una propuesta de intervención estructurada, destinada al uso en investigación y en formación de psicoterapeutas, dentro de una perspectiva de Terapia de Constructos Personales (TCP).

La Psicología de Constructos Personales (PCP) fue propuesta en 1955 por George Kelly, como una teoría general para la comprensión de la experiencia 
humana (Botella y Feixas, 1998; Fransella y Dalton, 1990). Esta teoría tiene como base epistemológica el alternativismo constructivo, según el cual hay varias alternativas de construcción posibles para dar sentido al mundo. Nadie tiene acceso directo a la verdad, sino que cada uno la ve desde su punto de vista personal. Para este autor, atribuimos significado al mundo a través de un sistema de constructos bipolares que creamos a lo largo de los años por asimilación y acomodación de la experiencia. Este sistema nos permite anticipar el significado de los acontecimientos, haciendo el mundo más previsible y, así, más seguro. Somos como "científicos personales", construyendo micro-teorías sobre todo con lo que nos encontramos. Día tras día conducimos experimentos para poner a prueba nuestras hipótesis: todo comportamiento es en sí mismo un experimento, una cuestión planteada de forma más o menos consciente. Si estos experimentos confirman nuestras hipótesis, la teoría es validada; si las hipótesis son desconfirmadas, deberá haber una remodelación. El aprendizaje ocurre en la reconstrucción sucesiva de los acontecimientos. El desarrollo humano es, entonces, un proceso continuo y cíclico de construcción de significados (Feixas y Villegas, 2000; Fernandes, 1993; Kelly, 1955).

\section{Principios de Terapia de Constructos Personales}

De acuerdo con la epistemología constructivista no es posible tener una interpretación correcta de la realidad; cada persona tiene su visión del mundo, que puede ser más o menos funcional. Aparece un problema cuando el sistema de construcciones del sujeto no es capaz de acomodarse a los acontecimientos con los que se enfrenta. Entonces, los constructos pueden ser invalidados continuamente, pero seguir siendo utilizados repetidamente (Kelly, 1955). Contrariamente a otros abordajes terapéuticos, que se centran en el síntoma y su eliminación, las perspectivas constructivistas (Feixas y Villegas, 2000; Neimeyer y Mahoney, 1995) se concentran en buscar los significados implicados en el problema, o sea, en comprender las construcciones que el cliente hace de sí mismo y del problema, y la forma como estas construcciones se relacionan con el propio problema.

Si para Kelly el ser humano es un científico, el psicoterapeuta es un asesor científico, que acompaña al cliente en sus experimentos, dándole control, limitando el número de variables y su complejidad (Tschudi, 1977). Utiliza la sala de terapia como un laboratorio (Kelly, 2001). Así, la evaluación constructivista se basa en una actitud crédula, en la que el cliente es considerado el mayor especialista en su propio sistema de construcción. Se busca conocer las dimensiones utilizadas por la propia persona para comprender y estructurar su realidad. El terapeuta necesita entender el punto de vista desde el que el cliente da solución a sus problemas. Tal solución, aunque sintomática, no es estúpida ni irracional, es la mejor que ha sido capaz de encontrar hasta el momento. Pero siempre hay una forma de construcción alternativa, que puede resultar más funcional, menos invalidante. 
La relación terapéutica se basa en una actitud colaboradora, "de experto a experto" (Botella y Feixas, 1998; Feixas, 1995). El terapeuta tiene una perspectiva curiosa, semejante a la del científico, en la que los constructos del cliente son hipótesis que deberán ser exploradas y testadas. Sin embargo, iniciar nuevos caminos puede dejar al cliente perdido y sin dirección - o sea, sin construcciones disponibles para su experiencia, lo que Kelly denomina ansiedad. Es importante dar al cliente la seguridad de que no está obligado a abandonar sus viejas construcciones (Tschudi, 1977).

A nivel técnico, la TCP hace algunas propuestas (Feixas y Villegas, 2000; Botella y Feixas, 1998), pero deja espacio a la utilización de técnicas diferentes, siempre y cuando se adecuen a una conceptualización constructivista del caso. Cada técnica suele ser propuesta al cliente y evaluada en conjunto, ya que el cliente es el investigador principal en el proyecto.

\section{Los dilemas implicativos}

La noción de dilema implicativo surge por primera vez en la Tesis Doctoral de Dennis Hinkle (1965) dirigida por el propio Kelly. Hinkle explica que "una posición polar en un determinado constructo determina posiciones polares en otros constructos" (p. 17). La expresión dilema implicativo la utiliza para referirse a una forma de implicación ambigua entre constructos, provocada bien por confusión entre contextos de utilización de los constructos o bien por utilización de la misma etiqueta para dos constructos diferentes.

Pero ya Kelly (2001) hablaba de dilemas en contraposición a la idea ya clásica de paradoja neurótica (acciones que realiza el cliente, o inhibiciones, que son perjudiciales para su desarrollo). Dice el autor que cuando un comportamiento no cesa a pesar de no ser recompensado, o incluso ser destructivo, es que el individuo no dispone de alternativas más adecuadas. "Dentro de su propio sistema de construtos, el cliente se encuentra ante un dilema, no ante una paradoja" (p. 70).

Posteriomente, Ryle (1979) define los dilemas implicativos como dicotomías falsas (ej. "o eres tranquila o eres sensible") que restringen las posibilidades de elección o implicaciones falsas (ej. "si soy alegre entonces seré artificial"), que inhiben el cambio. También Tschudi (1977) habla de dilema implicativo, cuando el cliente desea hacer un cambio en un constructo, pero hay otro constructo que imposibilita ese movimiento. El sistema está bloqueado, la persona está encallada o forzada a caminar en círculos. Es en este mismo sentido que utilizamos actualmente la expresión, teniendo en cuenta la forma como el sujeto se construye a sí mismo y como desea poder construirse, o sea, como define a su ideal. Estos datos nos informan acerca de áreas de satisfacción (cuando su "yo actual” y su "yo ideal" están en el mismo polo del constructo) y de insatisfacción del sujeto (cuando su "yo actual" y su "yo ideal" están en polos opuestos). Así, hablamos de dilema implicativo cuando el problema o un aspecto que el sujeto desea cambiar está 
fuertemente asociado a dimensiones positivas de su construcción de sí mismo. O sea, cuando encontramos una fuerte vinculación entre un constructo discrepante (en el que el sujeto desea cambiar) y un constructo congruente (en el que el cliente está satisfecho de su posición actual). Esta asociación significa que el cambio de polo en un constructo implica también el cambio en el otro. En este caso, el cliente puede no realizar el cambio deseado en un constructo determinado porque eso conllevaría un cambio indeseado en el otro constructo. Está bloqueado entre el deseo de abandono del problema y el de mantener sus implicaciones positivas (o evitar implicaciones negativas del polo deseado).

Intentar provocar el cambio en clientes que se encuentren en esta situación sería no solo difícil sino incluso indeseable. El cliente, sabiamente, se resistiría a cambiar, como una forma de proteger su identidad de una invalidación masiva (Sánchez y Feixas, 2001). En lugar de luchar contra la llamada "resistencia", al detectar los dilemas y trabajar con ellos validamos el sentido de autoprotección del sujeto y aumentamos la calidad de la relación terapéutica, obteniendo así un mejor punto de partida para buscar el cambio (Feixas et al., 2001). Al reconocer la coherencia de la posición del cliente salvaguardamos su dignidad, ya que excluimos una etiqueta de déficit o incompetencia de su parte. Por otro lado, si sabemos qué aspectos impiden al cliente cambiar, podremos finalmente dejar de luchar contra él, y realmente luchar con él para alcanzar sus objetivos de una forma que sea aceptable para la persona.

El dilema implicativo es un elemento transversal a los diferentes diagnósticos clínicos del DSM o otros sistemas de clasificación. Se trata de una dimensión de estructura y no de contenido, o sea, diferentes clientes con estructuras dilemáticas pueden presentar diferentes diagnósticos, en función del contenido (o etiqueta) de los constructos envueltos en dichos dilemas.

\section{Evaluación}

Los dilemas implicativos pueden ser identificados en la situación clínica de diversas formas, pero nosotros hemos desarrollado (Feixas et al, 2001; Feixas y Saúl, 2005) un método estructurado a partir de la Técnica de Rejilla (Feixas y Cornejo, 1996; Kelly, 1955). Este método consiste en la identificación de constructos congruentes (constructos en los cuales el sujeto puntúa el "yo actual" y el "yo ideal" de forma semejante) y de constructos discrepantes (los que el cliente coloca el "yo actual" y el "yo ideal" en polos diferentes). El dilema ocurre cuando hay una asociación (correlación Pearson, $r>0,35$ ) entre un constructo congruente y otro discrepante, en el sentido de que si se realizara el cambio deseado en el constructo discrepante se daría también un cambio de polo no deseado en el congruente.

Como ejemplo típico de un dilema implicativo podríamos tomar alguno de los casos investigados por Winter (1989) con problemas de ansiedad social. En uno de estos casos (véase fig. 1), en la rejilla aparece el constructo discrepante "tímido" vs. 
"sociable", en el que el "yo actual" se coloca en el primer polo y el "yo ideal" en el segundo, indicando así que la persona desearía realizar un cambio y que, por lo que respecta a este constructo se halla insatisfecha consigo misma. Por otro lado, cuenta con el constructo congruente "modesta" vs. "arrogante" en el que tanto el "yo actual" como el "yo ideal" se ubican en el primer polo. Así, la persona se siente satisfecha con ser "modesta" y no le gustaría un cambio de polo en este constructo. Pero el análisis de la rejilla nos aporta aun otro dato: ambos constructos correlacionan, es decir están asociados en el sentido de que un el cambio deseado en el constructo discrepante implicaría también un cambio, en este caso no deseado, en el constructo congruente. Es por ello que se denomina "dilema implicativo"; el cambio deseado conlleva implicaciones negativas y deviene así un dilema que podríamos formular así: "si dejo de ser tímido y me convierto en sociable dejaría de ser modesto para ser arrogante". O bien a la inversa: "Si quiero evitar ser arrogante debo seguir siendo tímido", aunque ninguno de estos dilemas puede expresarse verbalmente por parte del cliente, puede que incluso nunca haya pensado acerca ello. Forma parte de la manera en que tiene estructurado su sí mismo y su mundo interpersonal, lo que no quiere decir que sea consciente de ello.

Figura 1. Ejemplo de dilema implicativo

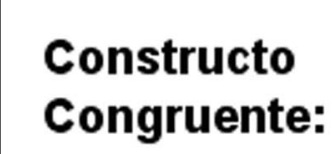

\section{Constructo discrepante:}

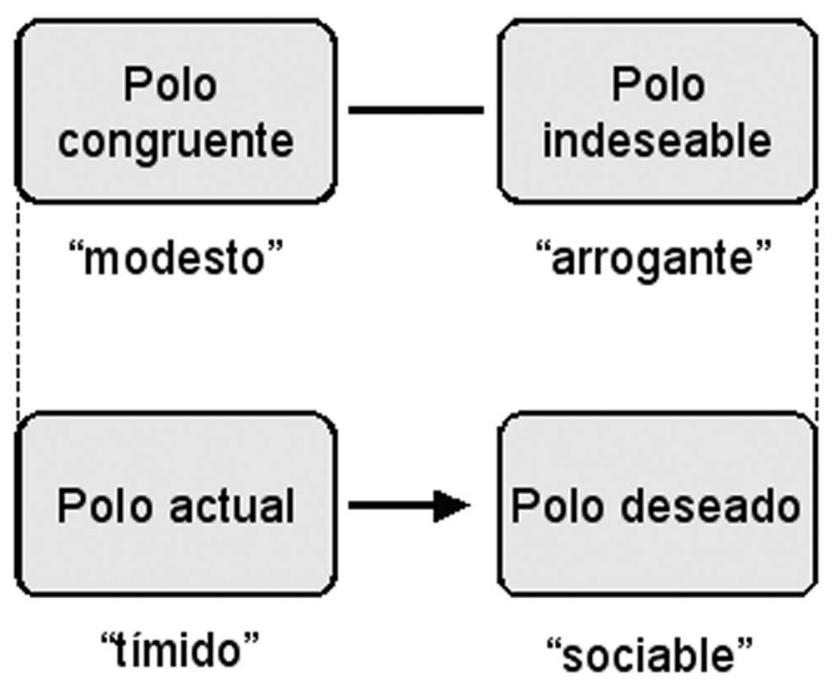

Analizando las rejillas de los clientes con el programa informático Record v. 4.0 (Feixas y Cornejo, 2002) se obtienen, además de otros datos de interés, las correlaciones entre todos los constructos, así como la identificación de los dilemas implicativos existentes. 


\section{El Proyecto Multicéntrico Dilema (PMD)}

En 1999 fue creado un proyecto de investigación, con la participación paulatina de diferentes centros de investigación en España, Portugal, Reino Unido, Italia y Sudamérica. Tiene el objetivo de estudiar el papel de los dilemas en diferentes problemas de salud (física y mental), así como desarrollar e implementar métodos terapéuticos focalizados en la resolución de estos dilemas. Los datos ya disponibles (Feixas y Saúl, 2004) indican que los dilemas pueden ser identificados en un tercio (34\%) de sujetos de una muestra no clínica de rejillas. En una muestra clínica (consultantes para psicoterapia), sin embargo, los dilemas se identifican en más de la mitad $(52,4 \%)$ de los sujetos. Los estudios ya realizados indican también que los clientes que no presentan dilemas al inicio de la terapia tampoco los suelen exhibir al final de la misma, mientras que más de dos tercios de la muestra que presentaba dilemas al comienzo de la terapia no los tenía al final. Estos datos sugieren que la psicoterapia, aunque no específicamente dirigida a la resolución de dilemas, produce una reducción significativa del número de dilemas implicativos (Feixas y Saúl, 2004; Saúl, 2005, Fernandes, 2007). Este proyecto se está desarrollando a diferentes niveles de trabajo, como la formación de investigadores, la creación de una base de datos de rejillas o el estudio de la frecuencia de dilemas en poblaciones específicas. "El último paso del proyecto es desarrollar un protocolo de intervención en dilemas implicativos con el objetivo de desarrollar e investigar el efecto de una Terapia Cognitiva Breve y Focalizada en el Conflicto Cognitivo" (Saúl, 2005). Este trabajo pretende desarrollar este último paso.

\section{Objetivos del manual}

Teniendo en cuenta los datos preliminares del PMD, parece claro que los dilemas, aunque no sean exclusivos de la población clínica, son más relevantes en estos casos, y tienden a desaparecer o reducir su número con el efecto de la psicoterapia, en paralelo con la reducción de la sintomatología. Estos datos han sido obtenidos con terapias psicológicas no orientadas al trabajo de los dilemas. Nos cuestionamos cuales serían los efectos de una terapia específicamente orientada a la solución de estos dilemas. ¿Será más eficaz un abordaje directo en la resolución de los dilemas? ¿Cual será la relación entre la resolución de los dilemas y la de la sintomatología? ¿Qué dificultades se pueden esperar en una psicoterapia focalizada en los dilemas implicativos? ¿Qué ventajas y desventajas tendrá este abordaje sobre los ya existentes?

Para responder a estas cuestiones se hace necesaria la creación de un manual de intervención breve y semi-estructurado, que facilite tanto la formación de terapeutas en este tipo de trabajo como el estudio del proceso y resultados de dicha terapia. Aunque la TCP defienda un eclecticismo técnico (siempre que se respete una coherencia teórica), a efectos de la investigación resulta necesario un guión de intervención que pueda ser aplicado de forma controlada, lo que exige mayor 
estructura y definición. Esto puede constituir también una ventaja para la formación de terapeutas noveles, que de esta forma podrán obtener mayor orientación para la práctica psicoterapéutica.

Este manual está basado en la propuesta original de Feixas y Saúl (2000), presentandose, sin embargo, de una forma más detallada y con opciones técnicas más limitadas. Proponemos una secuencia ya definida de estrategias con el fin de permitir una mayor uniformidad entre procesos terapéuticos. Para su elaboración ha también contribuido la participación de un grupo de trabajo en dilemas implicativos creado a este efecto en la Facultad de Psicología de la Universidad de Barcelona.

\section{Destinatarios}

Este manual puede ser utilizado con clientes adultos de consulta psicológica que presenten dilemas implicativos tal como son identificados con la TR. Asimismo, inspirados en la TCP y en consonancia con el interés de potenciar una buena alianza terapéutica, el cliente debe estar interesado en trabajar estos dilemas, aspecto que se va acordando en distintos momentos del protocolo. La falta de este interés por trabajar los dilemas por parte del cliente requiere suspender la aplicación del protocolo y centrar la terapia en los temas que obtengan el beneplácito del cliente, preservando así la calidad de la alianza terapéutica.

Este manual de terapia se puede aplicar a todo tipo de problemática o perturbación psicológica, exceptuando todo lo que se consideran trastornos mentales graves. En efecto, estas situaciones clínicas no podrían ser tratadas fácilmente con un protocolo tan corto y tan específico como éste. Sin embargo, nuestra propuesta podría quizá ser utilizada con estas poblaciones, como parte de una terapia más amplia, que cubra también otras áreas. Las situaciones de crisis deberán también ser excluidas, dado el carácter estructurado del manual y el hecho de que se centre en un aspecto cognitivo concreto y no siempre evidente para el cliente en un primer momento. Así, es previsible que en una situación de crisis el cliente no se encuentre disponible para analizar su estructura dilemática, por lo menos antes de solucionar cuestiones más pragmáticas y urgentes.

\section{Recomendaciones}

La aplicación de este protocolo deberá seguir los principios constructivistas en términos de relación terapéutica, evaluación y conceptualización de los problemas del cliente. Los terapeutas deberán tener formación constructivista y específicamente en las técnicas contenidas en el manual, incluyendo supervisión de los casos desde una perspectiva constructivista. El manual está pensado para sesiones semanales de una hora, con excepción de la fase 4 -la terapia de rol fijo-que está planeada para dos sesiones por semana. 


\section{Fases del manual}

1- Inicio del proceso

Las primeras dos sesiones se destinan al establecimiento de la relación terapéutica, así como a la recogida y elaboración del pedido de ayuda. En esta fase también se realiza la evaluación inicial, que además de la entrevista clínica y evaluación de la sintomatología, incluye la TR, instrumento constructivista que nos permitirá conocer un poco del sistema de construcción del cliente e identificar dilemas implicativos. En el caso de que no se hallen tales dilemas este protocolo no puede aplicarse.

2- Presentación de los dilemas al cliente

Esta fase tendrá, en principio, la duración de una sola sesión, la tercera del protocolo. Consiste en la devolución al cliente de los resultados de la evaluación inicial, centrándose especialmente en la exposición del dilema a trabajar.

3- Elaboración del dilema

Esta fase está compuesta por aproximadamente 5 sesiones, en que serán utilizadas diferentes estrategias. En consonancia con estas estrategias, están también previstas tareas que el cliente deberá realizar entre sesiones. Se pretende aumentar la comprensión que la persona tiene del dilema, tornándola capaz de identificar su influencia en situaciones concretas y, progresivamente, concebir formas de construcción alternativas, en las que el dilema no exista.

4- Resolución del Dilema

Solo llegarán a esta fase los casos en los que no se haya verificado ya una resolución de los dilemas y remisión de la sintomatología en la fase anterior. Cuando haya cambios significativos y éstos estén claramente implantados en la vida cotidiana del cliente, la terapia se terminará sin llevar a cabo esta fase.

Esta fase consiste en la utilización de una misma técnica, la Terapia de Rol Fijo (Kelly, 1955, véase Feixas y Villegas, 2000, para una descripción amplia y caso clínico) a lo largo de aproximadamente 5 sesiones. Esta técnica es complementada con algunas otras estrategias a utilizar puntualmente. El objetivo es que el cliente pueda ensayar en la práctica como sería vivir sin el dilema (o sin el problema), teniendo la seguridad de que no le será exigida su eliminación: si el cambio se le presenta como algo demasiado amenazador, podrá volver a su modo de funcionamiento habitual.

5- Finalización

Esta fase consiste solamente en una sesión, en la que se cerrará el trabajo con el protocolo y, posiblemente, el proceso terapéutico. Su localización en el protocolo depende de los resultados obtenidos, pudiendo tener lugar 
después de la $3^{\mathrm{a}}$ o de la $4^{\mathrm{a}}$ fase, según la necesidad de prolongar más o menos el trabajo con el cliente.

\section{Estructura de las sesiones}

Fase 1 - Inicio del proceso

$1^{\text {a }}$ sesión

\begin{tabular}{|l|l|}
\hline Objetivos & Estrategias \\
\hline $\begin{array}{l}\text { Establecimiento de la relación } \\
\text { terapéutica }\end{array}$ & Entrevista clínica \\
\hline $\begin{array}{l}\text { Reformulación de la demanda en } \\
\text { términos psicológicos operacionales }\end{array}$ & Análisis de la demanda \\
\hline $\begin{array}{l}\text { Evaluación de la construcción de } \\
\text { si mismo }\end{array}$ & Auto-caracterización \\
\hline \multicolumn{2}{|c|}{ Definición de objetivos para la terapia } \\
\hline
\end{tabular}

Evaluación inicial de sintomatologia

$2^{\mathrm{a}}$ sesión

\begin{tabular}{|l|l|}
\hline Objetivos & Estrategias \\
\hline $\begin{array}{l}\text { Acceder al sistema de construcción } \\
\text { del cliente }\end{array}$ & \multirow{2}{*}{ Técnica de Rejilla } \\
\cline { 1 - 1 } Identificar dilemas implicativos & \\
\hline
\end{tabular}

Fase 2 - Presentación de los dilemas al cliente

\section{$3^{\text {a }}$ sesión}

\begin{tabular}{|l|l|}
\hline Objetivos & Estrategias \\
\hline \multicolumn{1}{|c|}{ Devolución de los resultados de la evaluación } \\
\hline $\begin{array}{l}\text { Reformulación del problema en } \\
\text { términos de dilema }\end{array}$ & Presentación del dilema al cliente \\
\hline
\end{tabular}


Fase 3 - Elaboración del dilema

$4^{\text {a }}$ sesión

\begin{tabular}{|l|l|}
\hline Objetivos & Estrategias \\
\hline $\begin{array}{l}\text { Conocer las implicaciones supraorde- } \\
\text { nadas de los constructos que forman } \\
\text { el dilema }\end{array}$ & Escalamiento ascendente \\
\hline $\begin{array}{l}\text { Conocer las implicaciones subordina- } \\
\text { das de los constructos que forman } \\
\text { el dilema }\end{array}$ & Escalamiento descendente \\
\hline $\begin{array}{l}\text { Recuperar y detallar la formulación } \\
\text { del problema como dilema } \\
\text { Clarificar la definición de los objetivos } \\
\text { terapéuticos: encontrar alternativas } \\
\text { viables al problema }\end{array}$ & ABC de Tschudi \\
\hline
\end{tabular}

\section{$5^{\mathrm{a}}$ sesión}

\begin{tabular}{|l|l|}
\hline Objetivos & Estrategias \\
\hline $\begin{array}{l}\text { Concreción de la noción de dilema: } \\
\text { relación con la experiencia inmediata } \\
\text { del cliente }\end{array}$ & $\begin{array}{l}\text { Reconstrucción de la experiencia } \\
\text { inmediata en función del dilema }\end{array}$ \\
\hline
\end{tabular}

\section{$6^{\text {a }}$ sesión}

\begin{tabular}{|l|l|}
\hline Objetivos & Estrategias \\
\hline $\begin{array}{l}\text { Comprensión de las implicaciones } \\
\text { relacionales del dilema }\end{array}$ & $\begin{array}{l}\text { Exploración y análisis de las implica- } \\
\text { ciones relacionales del dilema }\end{array}$ \\
\hline
\end{tabular}

$7^{\mathrm{a}}$ y $8^{\mathrm{a}}$ sesiones

\begin{tabular}{|l|l|}
\hline Objetivos & Estrategias \\
\hline $\begin{array}{l}\text { Elaboración del dilema en una } \\
\text { perspectiva histórica de la génesis y } \\
\text { evolución del dilema }\end{array}$ & Reconstrucción histórica del dilema \\
\hline
\end{tabular}




\section{9a sesión}

\begin{tabular}{|l|l|}
\hline Objetivos & Estrategias \\
\hline $\begin{array}{l}\text { Elaboración de alternativas al } \\
\text { dilema }\end{array}$ & $\begin{array}{l}\text { Trabajo con las excepciones al } \\
\text { dilema y con las implicaciones } \\
\text { entre constructos }\end{array}$ \\
\hline $\begin{array}{l}\text { Integración de la experiencia del } \\
\text { dilema con los aspectos trabajados en } \\
\text { terapia hasta el momento }\end{array}$ & Redacción de la historia del dilema \\
\hline
\end{tabular}

Evaluación intermedia de sintomatología y de dilemas (nueva puntuación de la rejilla)

El terapeuta debe evaluar, en este momento, la necesidad de continuar con la terapia. En los casos en los que se verifiquen cambios claros y que hayan sido aplicados por el propio cliente en su vida cotidiana, se pasa directamente a la fase 5 de finalización.

\section{Fase 4 - Resolución del Dilema}

\section{$10^{\mathrm{a}}$ a $14^{\mathrm{a}}$ sesiones}

\begin{tabular}{|l|l|}
\hline Objetivos & Estrategias \\
\hline $\begin{array}{l}\text { Elaboración de alternativas al dilema } \\
\text { Experimentación asistida de actitudes } \\
\text { y comportamientos alternativos al } \\
\text { problema }\end{array}$ & Rol fijo del dilema resuelto \\
\hline $\begin{array}{l}\text { Integración del trabajo realizado a lo } \\
\text { largo de la fase } 4\end{array}$ & Carta al personaje del rol fijo \\
\hline
\end{tabular}

\section{Fase 5 - Finalización}

\section{$15^{\mathrm{a}}$ sesión}

\begin{tabular}{|l|l|}
\hline Objetivos & Estrategias \\
\hline \multicolumn{1}{|c|}{ Finalización del proceso terapéutico } \\
\hline Prevención de la recaída & $\begin{array}{l}\text { Anticipación de dificultades futuras } \\
\text { u obstáculos para el mantenimiento } \\
\text { de los beneficios y de formas de } \\
\text { afrontamiento }\end{array}$ \\
\hline $\begin{array}{l}\text { Valoración y consolidación de los } \\
\text { beneficios de la terapia }\end{array}$ & Revisión del proceso terapéutico \\
\hline
\end{tabular}




\section{Descripción de las técnicas}

Fase 1 - Inicio del proceso

Análisis de la demanda

El proceso terapéutico empieza, la mayoría de las veces, por la elaboración del pedido de ayuda - o de algo equivalente a una demanda (Kelly, 1955). Según Villegas (1996), esta demanda tiende a presentar-se en psicoterapia de forma semejante al pedido de ayuda médica, siendo frecuentemente necesaria su reformulación en términos psicológicos como condición preliminar para el trabajo psicoterapéutico.

a) Acogida de la demanda del cliente

b) Valoración de la demanda por parte del terapeuta

c) Cuando sea necesario, renegociación de la demanda en términos psicológicamente operacionales, que permitan un trabajo clara y abiertamente dirigido a dimensiones psicológicas del cliente, como es el trabajo con dilemas.

\section{Auto-caracterización}

"Si no sabes lo que pasa con una persona, pregúntaselo; es posible que te lo diga" (Kelly, 1955, p. 241). La técnica de auto-caracterización es presentada como una forma de hacer esa pregunta y así acceder a la construcción que el individuo hace de sí mismo. Es pedida al cliente con las siguientes instrucciones:

Me gustaría que escribieras una descripción de Fernando (nombre del cliente), como si fuera escrita por un amigo que lo conociera muy bien, tal vez mejor que nadie lo conoce en realidad. Escribe en tercera persona. Por ejemplo, empieza con: Fernando es...

Fase 2 - Presentación de los dilemas al cliente

Presentación de los dilemas al cliente

A continuación de una devolución de resultados de la evaluación inicial de la sintomatología, se centrará la atención sobre la rejilla del cliente.

a) Selección de los dilemas a trabajar

[Cuando haya más de un constructo discrepante implicado en dilemas; si no es el caso se pasa directamente a b)]

- Presentar al cliente los diferentes constructos discrepantes envueltos en dilemas;

- Preguntar qué importancia tiene cada uno de ellos: hasta qué punto desea cambiar en esa dimensión, y cuál es su relevancia para el problema actual o para su motivo de consulta.

- Preguntar si desea trabajar en terapia estas dimensiones y, si responde afirmativamente para más de una, por cuál desea empezar el trabajo. 
Ejemplo:

"Al leer tus respuestas en la rejilla, he notado que hay algunos aspectos en los que el 'yo actual' y el 'yo ideal' se encuentran bastante distantes, como por ejemplo tímido - sociable. El 'yo actual' es 'muy tímido' y el 'yo ideal' sería 'bastante sociable'. Parece que esta sería una dimensión en la cual desearías cambiar. ¿Es este un aspecto que te gustaría trabajar en la terapia?"

(repetir estas preguntas para los restantes constructos discrepantes envueltos en dilemas)

"De estos diferentes aspectos, ¿cuál te preocupa más en este momento? ¿Cuál te gustaría trabajar primero?

En el caso de que responda negativamente para todos los constructos, la terapia seguirá en otro formato, ajeno a este protocolo.

b) Exploración del constructo discrepante elegido

Explorar el significado de los polos actual y deseado.

Ejemplo:

"Para ti, ¿qué es ser una persona tímida? ¿Y qué significa ser sociable?"

Explorar otros constructos asociados.

Ejemplos:

“¿Cómo son, en general, las personas tímidas? ¿Y las personas sociables?"

"Las personas que son sociables, ¿qué otras características suelen tener? ¿Y las que son tímidas?"

Por supuesto, ésta no es una secuencia obligatoria; en el caso de que el cliente tenga dificultad en responder a alguna cuestión, se pasa a la siguiente.

c) Relación con los elementos de la rejilla: características asociadas ya referidas y otras (positivas e negativas).

Ejemplos:

"En la rejilla vemos que por ejemplo tu amigo Pedro es una persona 'muy sociable'. ¿Es esto así?"

"De hecho, también has respondido que él es 'muy alegre' y 'bastante gracioso'. Suena bastante agradable.” 


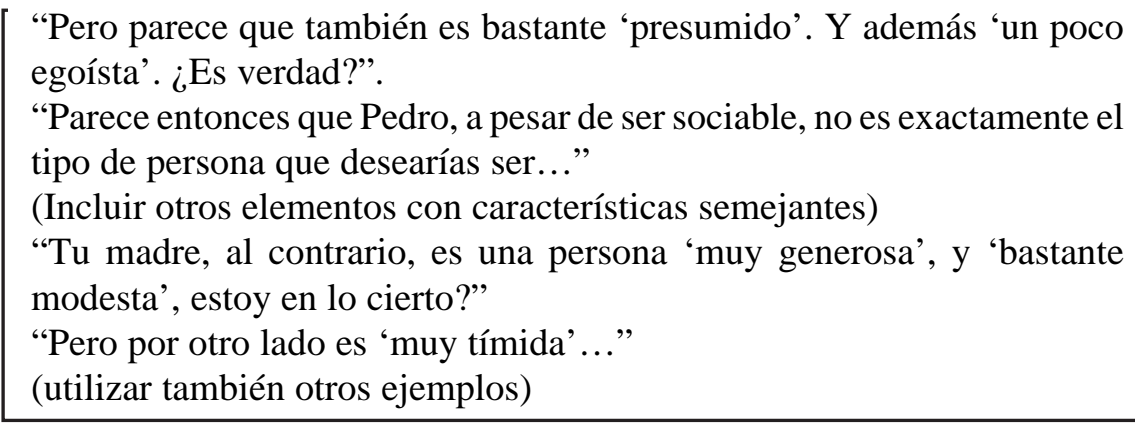

d) Presentación del dilema a partir de los ejemplos de las figuras exploradas: Ejemplos:

\begin{abstract}
"Realmente, teniendo en cuenta los ejemplos de estas personas que conoces, queda menos claro hasta que punto interesa ser sociable..."

"De hecho, las personas sociables que conoces tienden a ser también egoístas y presumidas. Y esto está muy lejos de tu ideal..."

"Por otro lado, las personas tímidas que conoces son en general modestas y generosas. Parecen más cercanas a lo que te gustaría ser..."

"Parece que estamos en una especie de dilema: deseas tornarte sociable, pero eso suele ir asociado a otras características que no te gustan nada. Así, casi que es mejor seguir siendo tímido, y por lo menos no perder aquellas características positivas que ya tienes, como generoso e modesto..." "¿Esto tiene sentido para ti?"

“¿Podría ser algo así lo que pasa contigo, y que hace que sea tan difícil superar la timidez?"
\end{abstract}

Proponer la focalización de la terapia en el trabajo con este dilema (por supuesto, en el caso de que el cliente haya aceptado esta reformulación).

Ejemplo:

“ ¿Tendría sentido para ti seguir trabajando acerca de este dilema, en la línea de lo que hemos hecho hoy?"

Si la persona no considera relevante trabajar esto, la terapia seguirá en otro formato, ajeno a este protocolo. 
Fase 3 - Elaboración del dilema

Escalamiento

Esta técnica ha sido creada por Hinkle (1965) con el objetivo de acceder a los constructos supraordenados de un constructo elicitado a un cliente o sujeto de investigación. El nombre escalamiento (laddering) fue propuesto posteriormente por Bannister y Mair (1968). Su flexibilidad, sencillez y su presunta capacidad para acceder a constructos más centrales (que se suponen más importantes) ha proporcionado gran popularidad a este método. Además, suele resultar muy interesante también para el sujeto que responde, ya que le proporciona una mayor comprensión de su propio mundo.

Neimeyer y sus colaboradores (2001) han realizado el primer estudio a gran escala $(n=103)$ destinado a evaluar la validez de constructo del escalamiento como medida de la estructura jerárquica de los sistemas de construtos, obteniendo cierto apoyo para asumir que la técnica de escalamiento ascendente efectivamente accede a dimensiones supraordenadas o nucleares de los sistemas de significado personales. Procede de la siguiente manera:

a) Tomar el constructo discrepante, y preguntar porqué el polo deseable es preferido al otro (polo actual).

b) La respuesta a esta cuestión constituye el peldaño siguiente de la escalera, siendo entonces necesario pedir el polo opuesto: el contraste a esa ventaja.

c) Teniendo presentes los dos polos, preguntamos cual de los dos lados prefiere la persona.

d) Una vez más preguntamos porqué, y cuál sería el contrario de esa ventaja. Tenemos así un peldaño más de la escalera.

Esta secuencia continúa hasta que la persona no sea capaz de explicar porque prefiere determinado polo, o solamente dé pequeñas variaciones de las respuestas dadas en niveles anteriores. El resultado es una jerarquía de varias capas de significados personales, como en el ejemplo:

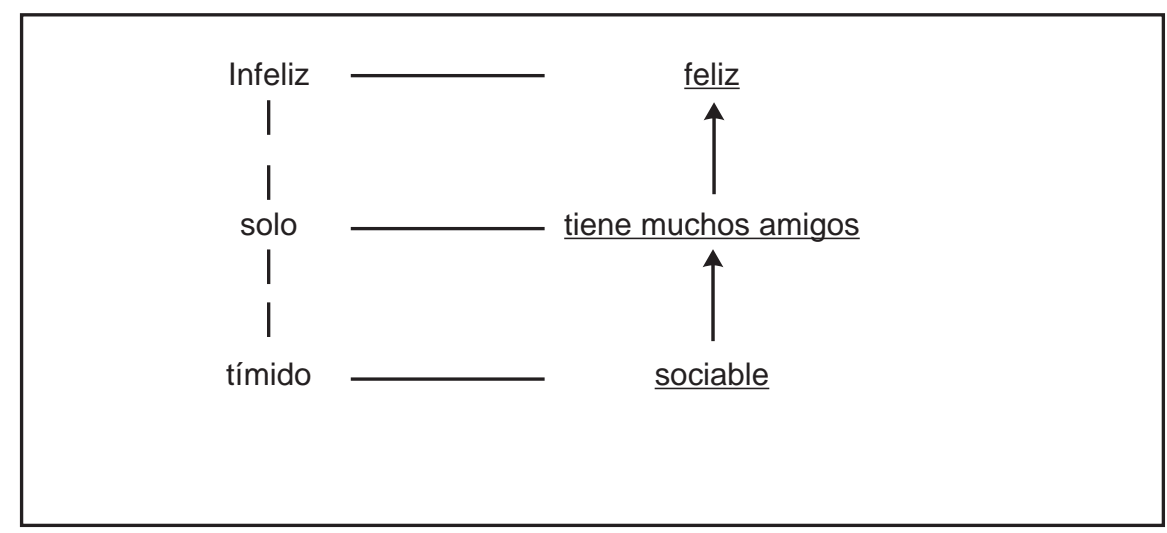


Escalamiento Descendente

a) Tomar el constructo inicial (discrepante) y pedir aspectos concretos del polo deseado, y sus opuestos.

Ejemplos:
"¿En qué se puede ver que alguien es sociable?"
“¿Cómo notas que alguien es sociable?"
“CCómo saben las personas que eres tímido?"
(para cada respuesta preguntar por el opuesto)

b) Preguntar, para los nuevos constructos, cual es la preferencia del sujeto. Si han surgido varios constructos subordinados, elegir uno para continuar el escalamiento, el que le parezca más importante al cliente o más relacionado con el problema).

c) Volver a pedir nuevos aspectos concretos, y sus opuestos.

Continuar el procedimiento hasta que los aspectos sean bastante concretos y/ o comportamentales, y por tanto no se pueda continuar buscando constructos subordinados. El resultado es una jerarquía descendente de significados personales, como en el ejemplo:

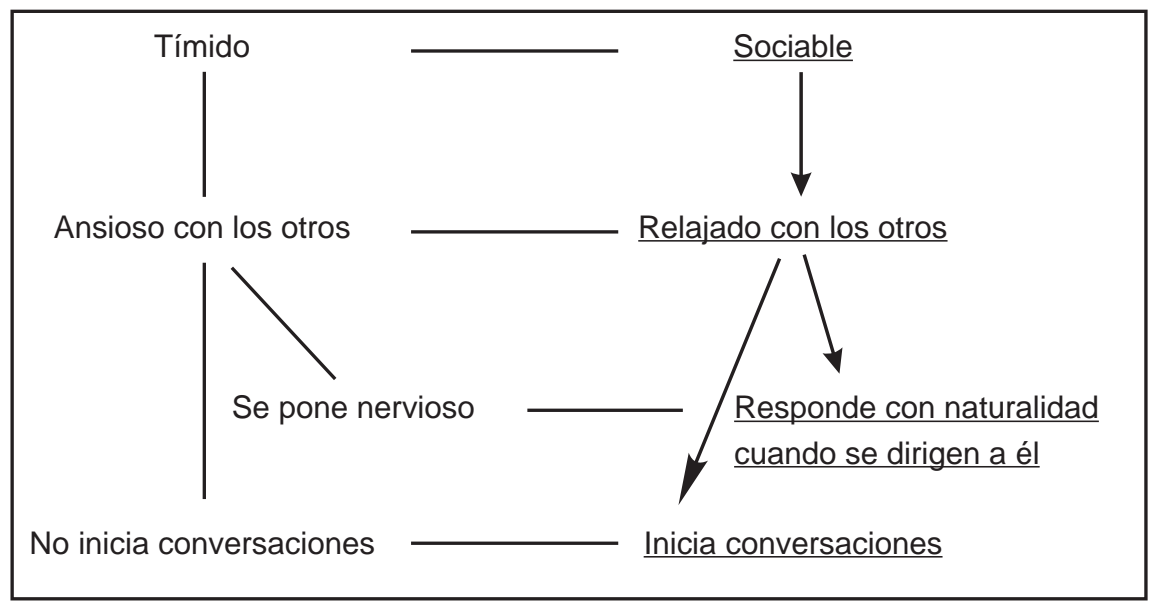

Repetir el procedimiento con el constructo congruente

Discutir con el cliente el ejercicio realizado: como lo ha vivido, aspectos que le han llamado la atención, descubrimientos realizados...; también aspectos que el terapeuta ha notado: dudas, constructos peculiares, cambios de lado de preferencia, constructos subordinados o supraordenados repetidos en los dos escalamientos...

Tarea para casa: reflexionar acerca del escalamiento, y responder a algunas cuestiones (el terapeuta deberá entregar al cliente una copia de la hoja del 
escalamiento y una ficha con cuestiones para reflexionar).

\section{ABC de Tschudi}

Desde una perspectiva kelliana, todo comportamiento es una pregunta. Tschudi (1977) defiende que los síntomas tienen muchas veces que ver con cuestiones mal formuladas, o formuladas de forma "poco honesta". Esta técnica ha sido propuesta con el objetivo de ayudar a las personas a clarificar sus objetivos y a plantear cuestiones más directas. Es una forma de representar un dilema implicativo, de clarificar que hay por lo menos un constructo que impide a la persona cambiar.

1- Tomar el constructo discrepante (A) (compuesto por los polos actual y deseado) y clarificar porqué esta situación es considerada un problema, o sea, qué ventajas hay en pasar al polo deseado, y que desventajas tiene el polo actual. Se obtendrán así los constructos B.

2- ¿Qué le impide a la persona cambiar? Para obtener los constructos $\mathrm{C}$, se puede pedir al cliente que piense acerca de las ventajas que puede tener el polo actual y las desventajas que puede tener el polo deseado.

3- Explicitar el objetivo de la terapia en función de este ejercicio: buscar otros medios para mantener las ventajas del problema que no el propio proble$\mathrm{ma}$ / síntoma, o una forma de combinar el polo deseado con las ventajas del problema.

Ejemplo:

\begin{tabular}{|c|c|c|}
\hline A: & Tímido & Sociable \\
\hline B: & $\begin{array}{c}\text { Dificultad en hacer amigos } \\
\text { Soledad }\end{array}$ & $\begin{array}{c}\text { Facilidad en hacer amigos } \\
\text { No sentirse solo }\end{array}$ \\
\hline C: & Auténtico & Artificial \\
& $\begin{array}{c}\text { No es rechazado } \\
\text { No se expone }\end{array}$ & $\begin{array}{c}\text { Riesgo de rechazo } \\
\text { Se expone }\end{array}$ \\
\hline
\end{tabular}

Reconstrucción de la experiencia inmediata en función del dilema

La reconstrucción es un concepto marcadamente constructivista, constituyendo la tarea principal de la psicoterapia (Harter, 1990). Tiene un papel central en el modelo de Guidano (1991) y, al mismo tiempo, es la última fase del ciclo de la experiencia (véase Feixas y Villegas, 2000), a través del cuál damos significado a la realidad. En esta técnica se revisa el ciclo de anticipación, implicación, encuentro, validación o invalidación y revisión constructiva que el cliente ha vivido en situaciones problemáticas. Se pretende ayudarlo a tomar consciencia de qué hipótesis está poniendo a prueba y qué resultados está obteniendo, así como a elaborar alternativas de construcción que resulten más válidas, al menos con más probabilidades de resultar validadas. Se procede de la siguiente manera: 
a) Tomar un episodio en el que aparece el problema y pedir al cliente que lo describa en detalle desde su inicio hasta la resolución.

b) Preguntar, en cada fase (contextualización, inicio de los acontecimientos, desarrollo de la acción, final del episodio), qué pensaba, qué sentía, qué ha hecho, qué ha pasado...

c) Identificar los puntos en los que se inician las emociones, y explorarlos en detalle:

a. Relacionar las emociones con los significados

b. Explorar las conexiones de la experiencia trabajada con el dilema: identificar elementos del constructo congruente en el episodio, explorar la vivencia de esos elementos, su relevancia, asociación con las emociones...

d) Elaborar alternativas al dilema en este episodio concreto (qué otras cosas se podrían pensar, que podría haber hecho...)

Tareas para casa: Recogida de nuevos episodios en que sea visible el dilema y, a su vez, episodios que constituyan excepciones el dilema.

\section{Trabajo con las implicaciones relacionales del dilema}

Dado el rol fundamental de las relaciones interpersonales en la formación de los significados personales, también en el origen y mantenimiento de los dilemas los otros significativos tienen un lugar de relieve. Así, pretendemos hacer que el cliente sea más consciente de cómo sus relaciones interpersonales determinan su construcción de la realidad, para que se vuelva capaz de crear construcciones alternativas, menos dependientes de aquellas figuras.

a) Tomar un acontecimiento en el que sea visible el dilema y pedir al cliente que lo describa con detalle.

b) Identificar en el episodio las figuras que validan o confirman el dilema (los "cómplices" del dilema), es decir, las personas que representan los polos deseado e indeseado. O bien aquellas que esperan que el cliente siga comportándose así, o que indican que si se diera el cambio deseado eso supondría un cambio más global e indeseado para el cliente, si las hubiese.

c) Buscar figuras alternativas al dilema, las que se encuentren al mismo tiempo en los polos deseado y congruente.

\section{Reconstrucción histórica del dilema}

El significado de la experiencia está ligado a la naturaleza y al proceso de construcción narrativa, así como a la coherencia de la misma narrativa. Al construir su propia historia, el individuo necesita mantener su sentido de identidad a través de las diferentes narrativas existentes a lo largo de su vida, así como en cada una de las narrativas que la componen (Gonçalves, 1998). La reconstrucción histórica permite comprender el sistema de construcción del cliente en diferentes momentos 
de su historia, y su coherencia a través del tiempo. Se pretende identificar en el tiempo el surgimiento del dilema y las diferentes formas de vivirlo a lo largo de los años, promoviendo en el cliente un sentido histórico de coherencia.

a) Pedir al cliente que recuerde, a lo largo de su vida, un episodio por año en que se verifique el dilema (siempre que sea posible; es natural que para algunos años el cliente no consiga identificar ningún episodio).

b) Verificar qué representaban los diferentes polos del dilema en aquel momento vital.

c) Después de la recogida de todos los episodios, discutir con el cliente: cómo el dilema ha tomado forma a lo largo del tiempo, qué momentos se asocian a su solidificación, qué figuras han sido importantes en esa construcción...

d) Tarea para casa: identificación de episodios a lo largo de la vida en que no ha estado presente el dilema.

\section{Elaboración de alternativas al dilema}

Incluso en las historias más saturadas por los problemas, hay momentos en los que estos no han dominado. Estas excepciones son el motor del cambio: en la medida que son identificados y explorados abren camino para una forma alternativa de construir la realidad, volviéndose cada vez más frecuentes (White y Epston, 1990; Gonçalves y Henriques, 2002).

a) Tomar las excepciones al dilema encontradas (en el trabajo anteriormente realizado, tanto en las sesiones como en casa)

b) Extender esas excepciones a otros momentos y contextos: ¿qué sería necesario para que la excepción ocurriera más veces? ¿Cómo podemos conseguirlo?

\section{Redacción de la historia del dilema}

Esta estrategia se basa en los presupuestos de la narrativa como principio organizador de la experiencia humana (Sarbin, 1986). La escritura acerca de los problemas de uno ha mostrado traer beneficios para la salud física y mental. Una explicación para este hecho es que escribir ayuda las personas a reorganizar sus pensamientos y sentimientos y a crear narrativas más coherentes o significativas sobre los acontecimientos de su vida (Graybeal, et al., 2002). Para este fin, se propone al cliente la redacción de la historia del dilema trabajado: cómo ha surgido, cómo ha influenciado su vida, cómo lo ve ahora.

Fase 4 - Resolución del Dilema

\section{Terapia de Rol Fijo}

La terapia de rol fijo es propuesta en la obra de Kelly (1955), pero ya venía siendo utilizada por él desde finales de los años 1930. Es considerada la técnica más 
distintiva de la TCP, y una forma practica y radical de conseguir el cambio (Neimeyer el al., 2003). Según Neimeyer y Bridges (2003, cit Neimeyer et al., 2003), esta ha sido la primera forma de terapia breve.

Consiste en un experimento proyectado por el terapeuta y que debe ser aceptable para el cliente, en el que crea un rol nuevo, un personaje ficticio que enfrenta desafíos semejantes en su vida, pero que los aborda desde una perspectiva diferente. Este rol alternativo es construido por el terapeuta como la caracterización de un personaje a partir de una auto-caracterización del propio sujeto (técnica de evaluación también propuesta en la obra de Kelly, 1955). El cliente representa este personaje por un período determinado de tiempo (normalmente algunas semanas), con el objetivo de probar como sería construir el mundo de una forma distinta a la habitual. Al final del experimento, terapeuta y cliente evalúan los resultados y éste decide si quiere o no mantener algunas de las características experimentadas.

\section{Presentación del esbozo del rol fijo}

a) Presentar al cliente el esbozo del rol fijo previamente preparado por el terapeuta.

b) Preguntar al cliente:

- Si este personaje le parece real;

- Si se parece a alguien que a él/ ella le gustaría conocer.

c) Hacer, conjuntamente con el cliente, los ajustes necesarios al esbozo.

\section{Proponer el desempeño del rol fijo:}

Ejemplo:

"En las próximas semanas, en lugar de tratar directamente tus problemas, me gustaría sugerir algo diferente. Supongamos que Fernando se va de vacaciones y en su lugar se quedará Pablo. Actuarás como Pablo, hablarás como él y hasta pensarás como él. Harás todo lo que haría él, comerás lo que comería él y, si es posible, tendrás los sueños que Pablo tendría. Quédate con esta copia del guión de Pablo y léela varias veces por día (especialmente por la noche y por la mañana, cuando te levantes), y siempre que tengas dificultad en desempeñar este rol.

Durante este tiempo nos veremos dos veces por semana, para ayudarte a desempeñar este rol, anticipando algunas situaciones para que puedas desempeñar el rol de Pablo lo mejor posible. No te preocupes con Fernando durante este tiempo, ni siquiera tenemos que pensar en él por ahora - está de vacaciones y no quiere ser molestado. Ya volverá de aqui a unas semanas y entonces podremos hablar con él."

3. Proponer los primeros ensayos en función de las actividades del cliente en los 
próximos días, priorizando interacciones profesionales o académicas.

4. Ensayar algunas de las situaciones en las que deberá poner en práctica el nuevo rol (role-playing).

Tareas para casa: Desempeño del rol fijo en situaciones de trabajo o académicas.

(En la sesión siguiente)

5. Evaluación del ensayo del rol fijo durante la semana anterior: éxitos y dificultades, reacciones de los otros, sentimientos...

6. Preparación de los próximos ensayos y anticipación de dificultades (se trabaja con role-playing para afrontarlos).

Repetir el procedimiento con los sucesivos niveles de ensayo: con compañeros y amigos del mismo sexo; con el cónyuge (o una persona del sexo opuesto); con los padres.

7. Cierre del rol fijo: evaluación de la experiencia del rol fijo a lo largo de las semanas anteriores - sentimientos, reacciones esperadas e inesperadas, ventajas y desventajas en relación al sí mismo habitual, aprendizajes... Decisión de qué pretende o no mantener/ aprovechar el cliente del rol fijo para su propia vida.

\section{Carta al personaje del rol fijo}

La escritura de una carta de despedida es habitual en el trabajo de algunos terapeutas constructivistas (Mahoney, 2003). Ésta es una estrategia de preparación del cierre del proceso terapéutico, en este caso del protocolo de trabajo con dilemas. Al mismo tiempo, surge en continuidad con la terapia de rol fijo, funcionando también como un cierre para esta actividad, ya que se dirige justamente al personaje encarnado durante ese experimento.

\section{Notas finales}

Este manual ha sido creado en el contexto de un proyecto de doctorado, constituyendo un primer paso en un estudio más extenso. A continuación, se formarán psicoterapeutas para su aplicación, y se tratarán varios casos de acuerdo con esta propuesta, recibiendo una supervisión adecuada al protocolo. Estos casos, su proceso terapéutico, serán objeto de un estudio intensivo, intentando responder a diversas cuestiones tanto a nivel de resultados como del propio proceso y relación terapéutica.

Este trabajo ha sido posible gracias a la ayuda recibida por Joana Senra del Programa Operacional Ciencia e Inovaçao 2010, Ministerio da Ciência, Tecnología e Ensino Superior, y por el Fondo Social Europeo de la Unión Europea. 
La idea de que los humanos tenemos conflictos internos y que de ellos se puedan derivar síntomas y/o sufrimiento es muy antigua en el campo de las psicoterapias. Sin embargo, pocos han sido los intentos por apreciar esta cuestión de forma empírica. Precisamente, el objetivo del Proyecto Multicéntrico Dilema ha sido investigar el papel que tienen en la salud estos conflictos, definidos como dilemas implicativos o constructos dilemáticos identificados a través de la Técnica de Rejilla. Se ha detectado una mayor prevalencia de estos conflictos en una gran variedad de problemas clínicos (depresión, fobia social, problemas somáticos, etc.), por lo que parece indicado desarrollar una forma de intervención que permita abordarlos y resolverlos.

En este trabajo se presenta un manual de intervención enfocado a la resolución de dilemas implicativos. Se trata de una propuesta de intervención estructurada, de 15 sesiones, destinada principalmente al uso en investigación y en formación de psicoterapeutas, dentro de una perspectiva de Terapia de Constructos Personales.

Palabras clave: Constructos Personales; dilemas; psicoterapia; Técnica de Rejilla

\section{Referencias bibliográficas}

Botella, L. y Feixas, G. (1998). Teoría de los constructos personales: Aplicaciones a la práctica psicológica. Barcelona: Laertes

Feixas, G. (1995). Constructos personales en la práctica sistémica. En R. A. Neimeyer y M. J. Mahoney (Comps.), Constructivismo en psicoterapia (pág. 273-309). Barcelona: Paidós, 1998.

Feixas, G., Ávila, A., Saúl, L. A. y Sánchez, V. (2001). Implicaciones terapéuticas de los conflictos cognitivos. Revista Argentina de Psicología Clínica, X(1), 5-13

Feixas, G. y Cornejo, J. M.(1996). Manual de la técnica de rejilla mediante el programa RECORD V. 2.0 (2ª ed.). Barcelona: Paidós

Feixas, G. y Saúl, L. A. (2000) Detección, Análisis e Intervención en Dilemas Implicativos. http://www.usal.es/ tcp/protoCastellano/introduccion.htm 26-06-2006

Feixas, G. y Saúl, L. A. (2004). The Multi-Center Dilemma Project: An Investigation on the Role of Cognitive Conflicts in Health. The Spanish Journal of Psychology, 7, (1), 69-78

Feixas, G. y Saúl, L. A. (2005). Resolution of dilemmas by personal construct psychotherapy. En David Winter y Linda Viney (Eds.), Personal Construct Psychotherapy: Advances in theory, practice and research. London: Whurr

Feixas, G. y Villegas, M. (2000) Constructivismo y Psicoterapia (3º ed. rev.). Bilbao: Desclée de Brouwer.

Fernandes, E. (1993). Exploração de Construtos Pessoais sobre o self em mudança: Um estudo preliminar com sujeitos depressivos e agorafóbicos. Dissertação de candidatura ao grau de Mestre em Educação. Braga: Instituto de Educação e Psicologia, Universidade do Minho.

Fernandes, E. (2007). When what I wish makes me worse... to make flexible the coherence. Psychology and Psychotherapy: Theory, Research and Practice, 80, 165-180.

Fransella, F. y Dalton, P. (1990). Personal Construct Couselling in Action. London: Sage

Gonçalves, M. y Henriques, M. R. (2002). Terapia Narrativa da Ansiedade. Coimbra: Quarteto

Gonçalves, O. (1998). Psicoterapia Cognitiva Narrativa: manual de terapia breve. Bilbao: Desclée de Brouwer, 2002.

Graybeal, A., Sexton, J. D. y Pennebaker, J. W. (2002). The Role of Story-Making in Disclosure Writing: The psychometrics of narrative. Psychology \& Health, 17, 571-581

Harter, S. (1990). La psicoterapia como proceso reconstructivo. Revista de Psicoterapia, 1, 57-70.

Hinkle, D. N. (1965). The Change of Personal Constructs from a Viewpoint of a Theory of Construct Implications. Tesis Doctoral no Publicada. The Ohio State University.

Kelly, G. (1955).The Psychology of Personal Constructs (2 vol.). New York: Norton. 
Kelly, G. A. (2001). Psicología de los constructos personales: Textos escogidos (B. Maher, compilación en inglés; G. Feixas, editor). Barcelona: Paidós.

Mahoney, M. J. (2003). Psicoterapia reconstructiva. Barcelona: Paidós, 2005.

Neimeyer, R. A., Anderson, A. y Stockton, L. (2001). Snakes versus ladders: a validation of laddering technique as a measure of hierarchical structure. Journal of Constructivist Psychology, 14, 85-105

Neimeyer, R. A., Ray, L., Hardison, H., Raina, K., Kelley, R. y Krantz, J. (2003). Fixed Role in a Fishbowl: Consultation-based fixed role therapy as a pedagogical technique. Journal of Constructivist Psychology, 16, 249-271

Ryle, A. (1979). The focus in brief interpretative psychotherapy: dilemmas, traps and snags as target problems. British Journal of Psychiatry, 134, 46-54.

Sánchez, V. y Feixas, G. (2001). La "resistencia" en la psicología de los constructos personales. Revista de Psicoterapia, 12 (46/47), 133-162.

Sarbin, T. R. (1986). The Narrative as a Root metaphor for Psychology. En Theodore R. Sarbin, (Ed.). Narrrative Psychology: the storied nature of human conduct. New York: Praeger

Saúl, L. A. (2005). El papel de los conflictos cognitivos en la salud mental: implicaciones para el cambio terapéutico. Tesis Doctoral no Publicada. Universidad de Salamanca.

Tschudi, F. (1977). Loaded and Honest Questions: A Construct Theory View of Symptoms and Therapy. En D. Bannister (Ed.), New Perspectives in Personal Construct Theory (pp. 321-349). London: Academic Press Villegas, M. (1996). El Análisis de la Demanda. Una doble perspectiva, social y pragmática. Revista de Psicoterapia, 26-27, 25-78.

White, M. y Epston, D. (1990). Medios narrativos para fines terapéuticos. Barcelona: Paidós, 1996. 